\title{
Deutungshoheit der Religionen?* \\ Weltanschauung und Wirtschaft im Horizont globaler Institutionen und in Auseinandersetzung mit der Ethik von Eilert Herms
}

\section{ANDRÉ MUNZINGER ${ }^{* *}$}

\section{Reflections on the Interrelationship of Religion and Economics in the Light of the Development of Global Institutions and with Regard to the Ethics of Eilert Herms}

What roles do and should religions play in globalised institutions? The ethical theory of Eilert Herms insists that religions are fundamental for understanding how societies function. His approach is based on phenomenological insights on the nature of religious experience. We examine his work and show bow from his theory of religion he develops a social theory in which politics, economics and science are dependent on the educational functions of religions and world-views. With regard to the challenges that globalisation presents this position attains strong relevance and requires careful analysis in comparing it with alternative theories. In sum it is argued that Herms breaks ground in conceptualising the salience of the pluralistic nature of those processes which form worldviews and hence societal goals. At the same time we show how his work needs to be differentiated by comparing it with other social theories and by clarifying the need to strengthen the role and potential of critical reflection.

Keywords: religion, economic and social ethics, fundamental ethics, institutions, globalisation.

\section{Einführung}

Ob Weltprobleme in Kyoto, Pittsburgh oder Rom verhandelt werden, ob Umwelt-, Finanz- oder Nahrungsmittelkrise, wiederholt stehen sich Verfechter zweier entgegengesetzter Positionen gegenüber: diejenigen, die auf die unsichtbare Hand einer ökonomisierten Globalisierung vertrauen, und diejenigen, die den Umverteilungs- und Regulierungskompetenzen des starken Staates das Wort reden. Welche Rolle aber spielen die religiösen Überzeugungen in diesen Verhandlungen? Sind die kulturellen, weltanschaulichen Bindungen entscheidende oder vernachlässigbare Größen der Weltpolitik? Während verschiedentlich die zunehmende Bedeutung der Religionen für globale Entscheidungsprozesse im 21. Jahrhundert hervorgehoben wird (vgl. z. B. Norris/Inglehart 2004/2007), wird indessen die Leistungskraft traditioneller Ethik grundsätzlich hinterfragt (vgl. z. B. Homann 2005). Schließlich erkennen Andere zwar

Beitrag eingereicht am 22.10.2009, nach doppelt verdecktem Gutachterverfahren überarbeitete Fassung angenommen am 17.06.2010.

** Dr. André Munzinger, Evangelisch-Theologische Fakultät der Rheinischen Friedrich-WilhelmsUniversität, Am Hof 1, D-53113 Bonn, Privat: Guldenbachstr. 5, 50935 Köln, Tel.: +49-(0)2217885369, E-Mail: andremunzinger@hotmail.com, Forschungsschwerpunkte: Fundamentaltheologie, Religionsphilosophie, Sozialethik, Entwicklungs- und Globalisierungsfragen. 
den Einfluss der Weltanschauungen an, plädieren aber für die Übersetzung derer Ansprüche in eine säkulare, allen zugängliche Sprache (vgl. z. B. Habermas 2009). Für Eilert Herms verrät dagegen bereits die Fragestellung weltanschauliche Interessen. Selbst die empirische Analyse der Rolle der Religionen sei von vorwissenschaftlichen Prämissen geleitet, die kategorialer und deshalb weltanschaulicher Natur seien. Welche Konsequenzen ergeben sich aus seiner Hervorhebung der Religionen für die Gestaltung einer (globalen) Wirtschaftsordnung?

Das Ziel dieses Aufsatzes ist es, die Wirtschaftsethik von Eilert Herms zu erörtern, ${ }^{1}$ da der Tübinger und evangelische Theologe Herms innerhalb der wirtschaftsethischen Diskussion eine profilierte Position bezieht: Er will nicht nur seine eigene sozialtheoretische Perspektive als eine dezidiert theologische verstanden wissen, sondern jedwede Theoriebildung auf weltanschauliche Überzeugungen zurückführen können. ${ }^{2}$ Auf diese Weise werden zum einen die Sozialwissenschaften notwendigerweise auf die Reflexion von Religion verwiesen und zum anderen wird die unhintergehbare Stellung der Religionen im Gesellschaftsgefüge postuliert: Wirtschaft und Politik werden in ihrer Abhängigkeit von dem eigenständigen Funktionsbereich der Religion bzw. Weltanschauung erläutert. Zu untersuchen ist, inwiefern Herms den Religionen de facto die Deutungshoheit über die gesellschaftliche Ordnung überträgt. Ich werde zunächst seinen Religionsbegriff (2) und daraufhin seine Wirtschaftsethik erläutern (3). Nachdem ich die Pointen und Probleme dieser Konzeption erörtert habe (4), wird sie anhand aktueller Fragen um die Globalisierungsgestaltung veranschaulicht (5).

\section{Der Religionsbegriff im Werk von Eilert Herms}

Kann es überhaupt einen allgemein anerkannten Begriff der Religion angesichts ihrer vielfältigen, besonderen Erscheinungen geben? Für manche ist in der Tat dieser Metabegriff zu undifferenziert und sie warnen davor, Religion als anthropologische Konstante zu verstehen (vgl. Feil 2007). Für Andere dagegen ist der Begriff zu differenziert; alleine die Definitionssuche verrate ,abendländische Herkunft“, denn nur dort, „wo eine Differenz besteht zwischen der Gesamtheit einer Kultur und ihrer Religion“, werde diese Begriffsbestimmung überhaupt durchgeführt (Sundermeier, 2007: 11).

Herms ist sich bewusst, dass kein Konsens über die Semantik von „Religion“ besteht: „Der Ausdruck wurde und wird von streitenden Intellektuellengruppen jeweils entsprechend ihrer kultur- und theologiepolitischen Interessen verwendet" (Herms 2004g: 286). ${ }^{3}$ Es sei ein notwendiges wissenssoziologisches Desiderat, diese „polemi-

Neben umfangreichen religionstheoretischen Studien (vgl. z. B. Herms 2003) hat er Aufsatzbände zur Sozialethik (z. B. 1991; 1995; 2007) sowie zur Wirtschaftsethik (Herms/May 1986ff.; Herms 2004a-f) und zu Recht und Politik (2008) veröffentlicht.

2 Im Vergleich mit anderen theologischen Ethiken unterscheidet sich Herms durch sein radikal pluralistisches, aber nicht rein kulturalistisches Theorieprofil (vgl. z. B. Mack 2004 oder Hübner 2009, die eher ein arbeitsteiliges Verhältnis zwischen theologischer Wirtschaftsethik und Sozialwissenschaften verfolgen). Seine Position ist bisher in der Frage nach den Möglichkeitsbedingungen globaler Ordnung nicht diskutiert worden.

3 Damit meint Herms z. B. die religionskritischen Positionen seit dem 18. Jh., die Religion als Defizitzustand des Menschen postuliert haben, aber auch die theologischen Fassungen, die den Begriff dem wahren Glauben entgegengesetzt haben. Herms verbindet psychologische Einsich- 
sche Zerfaserung“ der Semantik dadurch zu klären, dass der phänomenologische Gegenstandsbezug dargestellt wird (ebd.: 287). In Anlehnung an den Theorieentwurf Friedrich Schleiermachers schlägt er vor, Religion als vorwissenschaftliche Bewusstseinslage und präreflexive Vertrautheit zu begreifen. Ist dieses unmittelbare Selbstbewusstsein in unhintergehbarer Weise prägend für jedwede Reflexion der Wirklichkeit, ${ }^{4}$ wird somit ein Begriff von Religion entwickelt, der gerade für die Sozial- und Erfahrungswissenschaften zugänglich sein soll. Wichtig ist an dieser Stelle zweierlei:

Zum Ersten verwendet er Religion als semantisches Äquivalent von Weltanschauung, Dogma, Daseins- und Existenzverständnis. Die Begriffe bezeichnen alle „diejenige kategoriale Gewißheit“ über Verfassung, Ursprung und Bestimmung der Welt und des Daseins, ,welche für das Menschsein aufgrund seiner ursprünglichen Konstitution als Personsein wesentlich ist" (Herms 2004a: 11). Mögen in einer Weltanschauung Überzeugungen über Ursprung und Ziel der Welt nicht ausformuliert sein, sind sie aber implizit präsent (vgl. Herms 2004g). ${ }^{5}$ Dabei ist anzumerken, dass Herms diese phänomenologische Religionsdefinition mit funktionalen Aspekten verbindet. Religion biete zugleich „die Kriterien der Vorzugswürdigkeit von wählbaren Zielen und orientiert daher die Zielwahl aller Personen“ (Herms 2004a: 11). Zum Zweiten ist darauf hinzuweisen, dass Herms sowohl die Kontingenz aller religiösen Erfahrungen wie auch ihre inhärente Gewissheitsstruktur betont. Jede Person erfahre ihre Lebenswelt als gegeben (passiv konstituiert) und durch bestimmte Erfahrungen erschlossen. In solchen Erschließungsgeschehen werden laut Herms Einsichten über Sachverhalte oder andere Personen zugänglich, die bisher verschlossen waren, von nun an aber als zuverlässig oder gewiss gelten. „Ohne Gewißheit in diesem genauen Sinne (des Inneseins der Zuverlässigkeit eines ,Bildes“ seiner Situation) kann kein Handlungsautor eine Entscheidung treffen“ (Herms 1991: 64). ${ }^{6}$ Diese anthropologischen Prämissen sind das Fundament für sein Gesellschaftsbild: Die grundlegendsten Erfahrungen des Menschseins sind unverfügbar und identitätsbildend zugleich. Einerseits will Herms somit positivistische Positionen hinterfragen, d. h. fundamentalistische oder rationalistische Positionen, die ihr Verständnis der Welt mit der Erfassung einer objektiv erschlossenen Wirklichkeit gleichsetzen. Andererseits will er relativistische Standpunkte kritisieren, die ihre eigenen Gewissheiten nicht anerkennen.

ten F. H. Jacobis, die Kulturtheorie F. Schleiermachers, pragmatistische Motive und phänomenologische Grundfiguren E. Husserls und M. Heideggers.

$4 \quad$ Herms behauptet nicht, dass Religion selbst die Möglichkeitsbedingung von Erkennen und Handeln ist, sondern sie ist das „gewordene Innesein“ dieser Möglichkeitsbedingung (Herms 2003: 292).

$5 \quad$ Herms kritisiert somit diejenige Unterscheidung zwischen Religion und Weltanschauung, die in der Tradition Humes eine Differenz zwischen wissenschaftlicher Weltanschauung auf der einen und Religion auf der anderen Seite darstellen soll. Er behauptet, dass diese Dichotomie sich „dem Interesse an einer Denunziation und Ausklammerung der überlieferten volkstümlichen Religion“ aus dem öffentlichen Vernunftdiskurs verdanke (Herms 2004a: 10f.).

6 Gewissheit sei aber nicht der Ausschluss von Unsicherheit, denn auch im Fall von Unsicherheit werden Entscheidungen ,gesteuert von Gewißheit; nämlich von einer Gewißheit über entscheidungsrelevante Lücken im ,Bild“ der Handlungssituation“ (ebd.). 


\section{Die Sozialtheorie und Wirtschaftsethik von Eilert Herms}

Das Verhältnis von Ethik und Ökonomik ist umstritten. Konsens besteht für Herms darüber, dass Ethik und Ökonomik „nicht additiv“ zueinander treten, ${ }^{7}$ sondern „eine innere Einheit" bilden. „Strittig ist jedoch, ob Ethik der umfassende Problembereich ist, innerhalb dessen Wirtschaft ein besonderer Problem- und Anwendungsbereich neben anderen ist $[\ldots],{ }^{8}$ oder ob die Ökonomik den umfassenden Horizont bildet" (Herms 2005a: 1623). ${ }^{9}$ Herms vertritt in dezidierter Weise die erste Position, in der Ökonomie innerbalb eines ethischen Horizontes betrachtet werden muss. Für ihn ist es unspezifisch, wenn Wirtschaft „letztlich“ als die Verringerung des Spannungsverhältnisses „zwischen Bedürfnissen und knappen Mitteln“ verstanden wird (Bartling/Luzius 2008: 5), weil dann nicht deutlich wird, dass Wirtschaft nur bestimmte Verringerungsleistungen erbringt, ${ }^{10}$ die abhängig sind von politischen Rahmenordnungen, technischem Wissen sowie Überzeugungen und Zielen, die jeden Prozess der Verringerung orientieren. Diese Überzeugungen entständen in einem Ethos, einer gewohnten, institutionalisierten Form des Zusammenlebens, und würden in einer Theorie dieser Form kritisch reflektiert: der Ethik (vgl. Herms 2004b). Das Ethos werde weder durch die ökonomischen Prozesse noch durch die Rahmenordnungen der Politik noch durch die Erkenntnisse der Wissenschaft generiert, sondern alleine aus den Erfahrungen, die das grundlegende Lebensgefühl für das (höchste) Gute und Ganze konstituieren. Vornehmlich geschehe dies in Sozialisations- und Bildungserfahrungen, in denen eben die weltanschaulichen oder religiösen Überzeugungen entwickelt würden. Wie aber werden die verschiedenen Funktionen einer Gesellschaft in Verhältnis gesetzt?

Eilert Herms beschreibt den wirtschaftlichen Funktionsbereich als eine von vier Teilordnungen des menschlichen Interagierens. ${ }^{11}$ Die anderen Teilordnungen sind die politische (als exekutive, legislative und judikative Herrschaft), die wissenschaftliche (technisches Wissen im weitesten Sinne) und schließlich die weltanschaulich-religiöse (ethische Bildung; vgl. Herms 1991; 1995; 2004a-e). In einer Abbildung dargestellt ergibt sich folgende Interdependenz:

7 Damit fallen die Positionen weg, die Moral als Ressentiment (in Anlehnung an F. Nietzsche) oder als „Appelitis“ ansehen (Luhmann 1993). Es wird von Herms vorausgesetzt, dass Ethik als Konkurrent der Ökonomik auftritt. Ethik und Ökonomik ständen formal betrachtet in einem notwendigen Wechselverhältnis; nur in welchem inhaltlichen Verhältnis sie stehen, bleibe umstritten. Keine Form des Wirtschaftens agiere ohne ethische Prämissen und gesamtgesellschaftliche Ziele, ob utilitaristische, marxistische oder christliche.

8 Dazu zählt Herms die Positionen von u. a. Peter Ulrich, Arthur Rich und die seinige.

9 Entweder würde, laut Herms, dieser umfassende Horizont um ethische Gesichtspunkte erweitert (so Josef Wieland) oder Ethik würde als Teilgebiet eingeschlossen (so Bruno Molitor) oder jener umfassende Horizont würde alle traditionellen Formen von Ethik ersetzen (so Karl Homann) (vgl. Herms 2005a: 1623; vgl. die Anmerkung zu Homann in Fn. 17).

10 Ziel des Wirtschaftens ist für Herms „die Bereitstellung und Verteilung aller Mittel“, „,nicht nur für die Gewährleistung des Lebens und Überlebens der Gattung im Naturzusammenhang, sondern für die Gewährleistung eines guten Lebens" (Herms 2004b: 67).

11 Herms verwendet die Begriffe Funktionsbereich, Teilsystem und Teilordnung als Synonyme. 


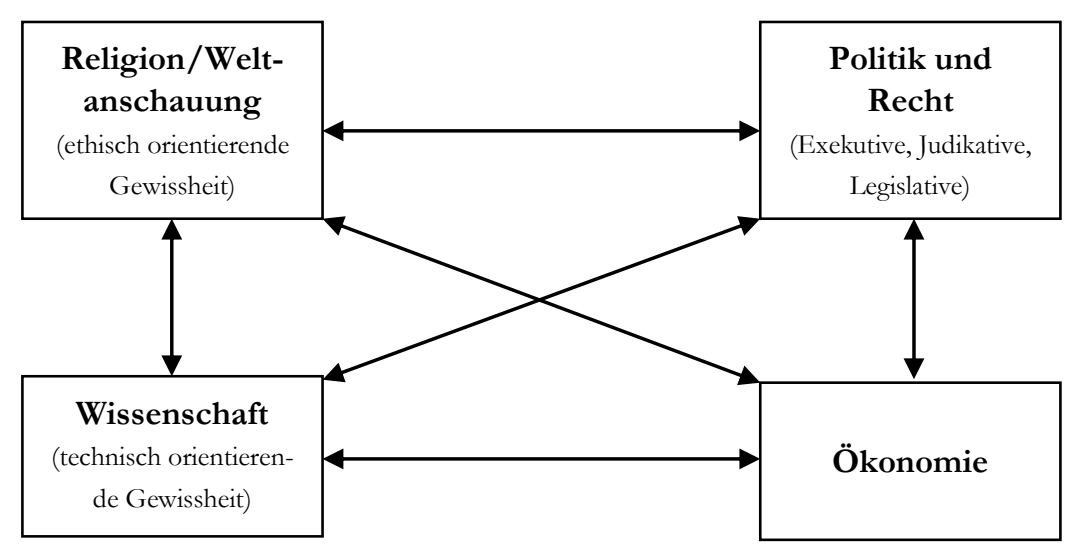

Abbildung 1: Gesellschaftsinteraktionsordnung (Herms 2004e: 242)12

Diese Teilordnungen sind nach Herms „gleichursprünglich“, aber in einer „natürlichen Hierarchie“ (Herms 2004e: 243): Die politische Interaktion ist fundamental, weil die Regelbefolgung dort sichergestellt wird, aber die Ziele dieser Regeln liegen schon in ethischen Überzeugungen vor, die in der religiös-weltanschaulichen Teilordnung erlangt werden. Gegenüber den politischen und weltanschaulichen Bereichen „haben die ökonomische und die wissenschaftliche Interaktion medialen Charakter. Die wissenschaftliche Interaktion erlaubt, Wege zu vorher feststehenden Zielen zu entdecken. ${ }^{13}$ Die ökonomische Interaktion erlaubt, solche Wege zu gehen“ (ebd.). Diese Funktionsbereiche vervielfältigen sich dann entsprechend in einer intergesellschaftlichen bzw. weltgesellschaftlichen Ordnung (Herms 2004e: 246; 2004f).

\section{Pointen und Probleme der Gesamtkonzeption von Eilert Herms}

An dieser Stelle werden die Theoriebereiche auf ihre Pointen und Probleme befragt und in der Abgrenzung zu konkurrierenden Entwürfen zugespitzt. Dabei sollte deutlich werden, dass die Moral-, Sozial- und Religionstheorie zwar selbstständig dargestellt werden, letztlich aber einen engen Zusammenhang bilden.

Zur Moraltheorie: Anders als kognitivistische, deontologische Moraltheorien stellt Herms die Ausrichtung am individuell erfahrenen (höchsten) Guten in den Mittelpunkt (vgl. Herms 2004d). Anders als andere teleologische Ethiken wird seine Güterlehre eingebettet in eine Pflichtenethik. Güter-, Tugend- und Pflichtenethik sind nur im Zusammenhang zu denken, da die Pflichten sich als Resultat bestimmter Güterabwägungen ergeben und die individuellen Wünsche sich wiederum nur mit Bezug auf das universal Gegebene realisieren lassen: nämlich der Teilnahme am institutionellen

12 Zu den genauen theoriegeschichtlichen Quellen dieses Schemas schweigt Herms, aber zur Auseinandersetzung mit Jürgen Habermas und für eine (ungenaue) Abgrenzung von Talcott Parsons vgl. Herms 1995: 251f.. Für den Abgleich mit weiteren Positionen vgl. Herms 2004a.

13 Allgemeine schulische Bildung erstreckt sich über die Funktionsbereiche Wissenschaft und Religion/Weltanschauung (vgl. Herms 2004f: 263). 
Gesamtgefüge. ${ }^{14}$ Diese Verschränkung von persönlichen Zielen (Überzeugungen, Gütern) und den universalen Möglichkeitsbedingungen ihrer Erfüllung ist grundlegend für seine Sozialtheorie. Inwiefern aber ist dann die Begründung zirkulär? Dass aus anderen inhaltlichen Überzeugungen andere Kategorien entwickelt werden, ist in der Tat im Hermsschen Ansatz angelegt. Der kategoriale Begriff ist zwar „der Form nach" inhaltlichen Überzeugungen übergeordnet - so Herms in der Deutung Schleiermachers -, aber „der Sache nach“ ist die kategoriale Theorie nur die abstrakte Darstellung einer inhaltlich geprägten konstitutiven Weltanschauung: „Die begriffliche philosophische Theorie bringt lediglich Züge zur Darstellung, die in der geschichtlichen Anschauung einer geschichtlich bestimmten Gestalt des ethischen Lebens impliziert sind" (Herms 2003: 121). Herms postuliert demnach, dass die Zirkularität in jeder Ethik vorkommt, und er möchte seine Konzeption so verstanden wissen, dass er seine weltanschaulichen Prämissen offenlegt, während Andere diese Klärung verweigern.

Lässt sich diese Sicht der Moral von einer kantischen Pflichtenethik ebenso wie von einer utilitaristischen Ethik abgrenzen, stellt Herms sie selbst in die Tradition klassischer Zugänge, die sich am „Sein“ des Einzelnen und des Kosmos ausrichten. Demnach ist zwar eine Orientierung am Nutzen sinnvoll oder sinnvoller als am abstrakten „Sollen“ (Herms 2004f: 254). ${ }^{15}$ Dezidiert fordert Herms aber die Unterscheidung der Arten des Nutzens ein. So stellt sich je nach Funktionssystem und je nach Güterrangfolge eine andere Gestalt (andere Ziele und Mittel) des Nutzens ein. Die Differenzen je nach Funktionsbereich ergeben sich aus den inhärent pluralverfassten Gütern, die Unterscheidungen hinsichtlich der Rangfolge aus der Vielgestalt der Präferenzen erfordern. Das „Sollen“ ist lediglich die Umsetzung des „Seins“, ${ }^{16}$ nämlich der verschiedenen Güterspektren, orientiert am jeweiligen Lebenssinn. Diese verschiedenen, nicht aufeinander zu reduzierenden Ebenen des Pluralismus aufzuzeigen, ist ein wesentlicher Beitrag von Herms zur Erfassung des komplexen, begehrenden Menschen mit diversen Kultur- oder Ethikaufgaben.

Zur Sozialtheorie: Politik und Wirtschaft sind notwendigerweise verwiesen auf weitere Funktionsbereiche, sonst - so Herms - unterliegen sie der Selbsttäuschung und Überforderung. Er weist auf, dass jede Form der Ökonomie und Politik von Voraussetzungen lebt, die sie selbst nicht generieren kann. Alle Überzeugungsbestände werden nur in manifesten Gewohnheiten und Institutionen kommuniziert, die weder ökonomisch erzeugt noch politisch garantiert werden können. Deshalb rücken auch politische Verordnungen in den Hintergrund der basalen Sozialisationsleistungen und Bildungseinrichtungen einer Gesellschaft, in denen diese Überzeugungen geformt und aufgebaut werden. Anders als Theorien, die in der Ökonomik die bessere Ethik se-

In Anlehnung an Friedrich Schleiermacher (vgl. Herms 2003) widerspricht er kantisch inspirierten Moraltheorien, in denen Moral und Ethos, Gutes und Gerechtes getrennt werden (Ulrich 2008: 30ff.).

Eine Einordnung dieser Position vor dem Hintergrund des breiten Spektrums utilitaristischer Differenzierungen steht noch aus.

16 Selbstverständlich muss Herms danach befragt werden, was das „Sein“ ist. Entbrennt an dieser ontologischen Prämisse zu Recht die Kritik der Vernunft, bleibt es ebenso unbefriedigend, wenn diese Vernunft sich aller Partikularismen zu entledigen sucht. 
hen, ${ }^{17}$ argumentiert Herms, dass sich die Ziele und Rahmenbedingungen jeder Ökonomie aus weltanschaulichen Quellen speisen. Veränderungen einer Wirtschaftsordnung können weder durch das Funktionssystem der Politik noch der Wirtschaft erzielt werden. Da das Wirtschaftssystem nur eine mediale Funktion gegenüber den anderen Teilordnungen einnimmt, darf der Wirtschaft die Leistung der "gesellschaftlichen Gesamtsteuerung" nicht auferlegt werden (Herms 2004e: 245).

Eine ausführliche Bewertung dieser Sozialtheorie müsste gegensätzliche Aspekte berücksichtigen: Einerseits kann zugestimmt werden, wenn Herms reduktionistische Konzeptionen hinterfragt: Demnach sind ethische Ansprüche, die z. B. eine gerechte Güterverteilung in einer Wirtschaftsordnung verlangen (wie wirtschaftskritische kirchliche Positionen), verkürzt. Sie müssten ihre Vorstellungen in ein Verhältnis setzen zu den anderen Teilsystemen einer Gesellschaft (vgl. ebd.: 248). Ebenso verkürzt sind Gesellschaftstheorien, die gesellschaftliche Ziele politisch planen und ökonomisch erzeugen wollen, ohne die weltanschaulichen und religiösen Institutionen zu berücksichtigen. Andererseits besteht die Gefahr der Ethisierung der Sozialtheorie: Dies betrifft weniger Herms' Ausgangspunkt im methodologischen Individualismus ${ }^{18}$ als vielmehr sein Rationalitätsverständnis. Die formale Einheit der Vernunft hat ihre Bedeutung nur innerhalb der materialen weltanschaulichen Überzeugungen (Herms 2005b: 1039f.). Infolge ist fraglich, ob und wie ein rationaler Diskurs zwischen verschiedenen Weltanschauungen mit der Möglichkeit wechselseitigen Lernens und gegenseitiger Kritik durchgeführt werden kann. Jedenfalls sind Argumente und Gründe als unabhängig von Erschließungsgeschehen zu bestimmen.

Zur Religionstheorie: Anders als Gesellschaftsordnungen, die der Religion eine private Nebenrolle zuordnen, stellt Herms diese als unhintergehbare Größe in den Mittelpunkt. Herms meidet ganz bewusst den Begriff der Zivilgesellschaft, weil sie eine illusionäre weltanschauliche Homogenität vortäuscht und die tatsächlichen Werte und Ziele (über Ursprung, Verfassung und Bestimmung des Menschseins) der Akteure nicht hinreichend thematisiert (Herms 1995: 256ff.). Dagegen ist kritisch einzuwen-

Herms grenzt sich von Karl Homann ab, der den systematischen Ort der Ethik in der Ökonomie und Marktwirtschaft als die institutionalisierte Form der Solidarität - unter der Bedingung eines besseren Marktes und geeigneter Rahmenordnungen - beschreibt (vgl. Homann 2005; Herms 2004d). Homann hat wiederum Herms vorgeworfen, zentrale Probleme, nämlich den Wettbewerb und das Gefangendilemma, nicht zu erwähnen (Homann 2006: 13). Meines Erachtens hat sich Homann wiederum mit der zentralen Hermsschen Anfrage nicht auseinander gesetzt, nämlich der Perspektivität seiner Kategorien. Diese Diskussion muss an anderer Stelle vertieft analysiert werden. Vgl. auch die Behauptung in der Studie von Lütge (2007), dass die Homannsche Ordnungsethik eher mit kulturübergreifender Zustimmung rechnen könne. Er plädiert dafür, das Eigeninteresse nicht zu beklagen, sondern als Chance zu sehen, „ohne Heuchelei, ohne Engstirnigkeiten und ohne moralistische Brillen, die den Blick für tatsächliche Problemzusammenhänge verstellen“ (261). Für einen ersten Vergleich zwischen Ulrich, Homann und Herms siehe Grabenstein (1998).

18 Seine Handlungstheorie läuft Gefahr, ihren individuellen teleologischen Bezugsrahmen zu überziehen: Die Diskussion zum unintendierten und kollektiven Handeln müsste berücksichtigt werden. Wenn Herms nämlich davon ausgeht, dass die ethischen Organisationen „das Verhalten der einzelnen Mitglieder in allen Interaktionsgebieten ihres Alltags innerlich steuern“ (Herms 1995: 255), wird diese Annahme selbst von Handlungstheoretikern bestritten oder differenzierter ausgeführt (zur Diskussion z. B. Joas 1996: 218-269). 
den, dass er unter Religion und Weltanschauung so unterschiedliche Bewegungen und Güter subsumiert, dass die Begriffe völlig überfrachtet werden. ${ }^{19}$ Zudem muss die Rückfrage an Herms bezüglich der von ihm eingeräumten zentralen Stellung der Religionen in der Gesellschaft bedacht werden:

Impliziert diese Bestimmung einen neuen Herrschaftsanspruch der Religionen oder Weltanschauungen (vgl. Tofall 2005)? Zwei Gründe können zunächst genannt werden, anhand derer die Frage verneint werden kann. Zum einen ist es kennzeichnend für Herms' Konzeption, dass es faktisch keine einheitliche ethische Position gibt. Denn jeder Mensch sei ausgerichtet auf Gutes, welches individuell erlebt, intersubjektiv begriffen und in Güterordnungen organisiert werde. Die Vielfältigkeit der individuellen und kulturellen Muster von Güterspektren und Präferenzen könne grundsätzlich „nicht zentral geplant werden. Vielmehr hänge sie vom polyzentrischen Zusammenspiel einer unendlichen Menge einzelner Überzeugungen und Entscheidungen ab“ (Herms 2004b: 55, 155). Für ihn steht diese Positionalität allen Handelns im Mittelpunkt: „Eine aperspektivische Ethik gibt es nicht“ (ebd.: 56). Das bedeutet dann, dass es nicht zu einer Monopolstellung einer Weltanschauung oder Religion, sondern nur zur Anerkennung der Vielfalt der ethischen Konzeptionen kommen darf (vgl. Herms 2004e: 244). Zum anderen ist es für Herms entscheidend, dass jede Teilordnung (Wirtschaft, Politik, Wissenschaft und Religion) sich auf die Erfüllung ihrer Funktion „unter Wahrung der selbständigen Erledigung der anderen Grundfunktionen durch die Organisationen und Sachwalter jener Bereiche“ beschränkt (Herms 2004f: 280). Somit wird klargestellt, dass alle Funktionsbereiche subsidiarisch ausdifferenziert werden müssen und Religionen und Weltanschauungen nur auf Kosten der eigenen Bestimmung in andere Ordnungen eingreifen. Infolge beinhaltet diese Konzeption einen religionskritischen und -begrenzenden Aspekt, insofern Herms voraussetzt, dass Religionen diese Ausdifferenzierung beachten müssen. Probleme entstehen laut Herms demnach nicht nur, wenn ein Funktionsbereich in die anderen Bereiche eingreift, sondern auch, wenn das Gesamtgefüge einer Gesellschaft von der Monopolstellung einer Weltanschauung bestimmt wird. Ohne den konsequenten weltanschaulichen Pluralismus sei das Ganze der Gesellschaft gefährdet.

Dennoch liegt eine Übergewichtung weltanschaulicher Überzeugungen vor. Letztendlich bleibt nämlich für Herms das wechselseitige Verhältnis zwischen den Funktionsbereichen „asymmetrisch“, weil die „radikalste Bedingungsdimension sozialer Evolution" in den individuellen Erschließungserfahrungen liegt (Herms 2009: 681). Damit relativiert Herms die Korrektivfunktion durch die Rationalitäten der anderen Funktionsbereiche. Wichtiger noch, die von ihm erwünschte Balance der Teilordnungen droht durch eine Immunisierung der Ethik und des Funktionsbereichs der Religion hinfällig zu werden.

Dass weltanschauliche und religiöse Institutionen im weitesten Sinne verstanden werden, zeigt sich an der - zu Recht höchst umstrittenen - Einordnung der sportlichen und künstlerischen Organisationen in den weltanschaulichen Funktionsbereich. 


\section{Anwendung auf die Diskussion um die Gestaltung der Globalisierung}

Die verstärkt zunehmenden internationalen, ökonomischen Interdependenzen werden vor dem Hintergrund einer tief greifenden Problematik eingeordnet: Wenn sich in den 1990er Jahren die Globalisierungskritik auf die wirtschaftliche Vernetzung begrenzt hat, so wird diese mittlerweile als Engführung verstanden, weil damit die Tiefendimension der Verflechtungen nicht erfasst worden sei. ${ }^{20}$ Zum einen ist bemerkt worden, dass auch die wirtschaftliche Vernetzung ohne politische Globalisierung gefährdet ist. So könne die Globalisierung von Produktion, Handel und anderen ökonomischen Märkten nicht effektiv bleiben, ohne eine korrespondierende Globalisierung von institutionellen Garantien der individuellen Freiheit und der Rechtsstaatlichkeit (Sautter 2004: 40). ${ }^{21}$

Zum anderen wird darauf hingewiesen, dass auch bestehende institutionelle Garantien gefährdet sind, weil der Weltmarkt politisches Handeln verdrängt und zugleich den Weltrisiken nicht gewachsen ist (Beck 2007: 13-55). Die „Global Player“ würden politische, soziale und ökologische Ordnungen unterlaufen, zugleich seien nationale Ordnungen diesen globalen Akteuren nicht gewachsen (so erneut Giddens 2007; Held/Kaya 2008). Ohne internationale Rahmenbedingungen schreite die „Kannibalisierung“" sozialer Identitäten, ökologischer Balance und weltweiter Ressourcen unaufhörlich voran (Radermacher 2005: 20). Jürgen Habermas verdichtet die Problematik und den Handlungsbedarf: Angesichts der Asymmetrien zwischen unterentwickelten und industrialisierten Ländern in „einer stratifizierten Weltgesellschaft" scheinen sich „unversöbnliche Interessensgegensätze zu ergeben“, wenn „keine institutionalisierten Verfahren transnationaler Willensbildung“ eingesetzt werden (Habermas 2000: 340). In diesem Sinne wird intensiv diskutiert, wie die zentrale Herausforderung bewusst gestalteter Globalisierung weltgesellschaftlich ausgerichteter Ordnungspolitik überhaupt bearbeitet werden kann (für einen Überblick vgl. Nuscheler/Messner 2006). Die Interdependenzen verlangen, so Volker Rittberger, nach neuen Ideen des „Weltregierens“, denn es herrsche eine Diskrepanz zwischen der Reichweite der Probleme und der politischen Lösungsinstrumente..$^{22}$ Wie aber das neue Souveränitätspara-

„,Globalisierung“ ist der Vorgang, daß Organisationen ausdifferenzierter Funktionsbereiche einer Gesellschaft auch in anderen Gesellschaften nach den Leistungsprinzipien ihrer Herkunftsgesellschaft tätig werden und damit Ansätze einer intergesellschaftlichen, tendenziell einer globalen Ordnung schaffen. Dieser Vorgang ist alt, nimmt aber gegenwärtig als wissenschaftliche, technische, mediale und wirtschaftliche Globalisierung rasantes Tempo an" (Herms 2004f: 268).

21 Gegen negative Bewertungen der Globalisierung müssen die positiven Auswirkungen z. B. mit Bhagwati (2007) hervorgehoben werden. Er stellt dar, wie China durch die Integration in den Weltmarkt die Armut um 28\% der Bevölkerung von 1978 bis 1998 verringern konnte. Auch Bhagwati ist für eine politische Gestaltung der Globalisierung, allerdings in erheblich liberalerer Weise als sein Hochschulkollege Joseph Stiglitz (2008).

Rittberger (2003: 180ff.) stellt dabei die verschiedenen Optionen dar: (i) Weltstaat: Dieser sei realitätsfern und beinhalte eine normative Problematik (190). (ii) Welthegemonie: Diese sei effektiv, aber habe eine selektive Realitätswahrnehmung und sei dazu normativ geschwächt (191). (iii) Weltweite horizontale Politik-Koordination: Diese sei relativ realitätsnah und normativ hinlänglich, aber in der Effektivität nur mäßig (194). (iv) Dazu gäbe es aber auch ein ganz neues Souveränitätsparadigma, welches den Staatszentrismus für tot erkläre (198f.). Dieses Paradigma sei aber selbst noch numinos und normativ problematisch. 
digma aussehen wird, bleibt freilich höchst umstritten. Einerseits ist der Nationalstaat eine „Schlüsselinstanz“ (vgl. Sassen 2008), andererseits ist das „Beharren auf Souveränitätsrechten" der Nationalstaaten nicht frei von Ironie, denn die Globalisierung hat diese Rechte „längst unterhöhlt“ (Sautter 2004: 210). Diese Ambivalenz hat sich in der Finanzmarktkrise (der Jahre 2008, 2009) in zugespitzter Weise manifestiert, weil für die notwendige internationale Regulierung keine entsprechenden institutionellen Vorkehrungen getroffen worden waren.

Aus dieser kurzen Analyse erscheint die Feststellung beinahe trivial, dass das globale Wirtschaftssystem alle anderen Gesellschaftsbereiche überlagert und politischer Regeln und Legitimation bedarf. ${ }^{23}$ Dass diese Einschätzung nicht basal ist, wird daran deutlich, dass internationale Regulierung unzulänglich und halbherzig bleibt. Sind die Gründe nun alleine im mangelnden politischen Willen und den nationalen Souveränitätsängsten zu suchen? Oder liegt die Problematik der (Wieder-)Herstellung des Primats der Politik auch darin, dass unter Vorstellungen von ,politischer Regulierung ${ }^{6}$ der Weltwirtschaft unspezifische Ziele subsumiert werden? Es ist in der Tat nicht unproblematisch, wenn das ,Primat der Politik' gefordert wird, denn die schlichte Dominanz von Staatlichkeit wird keine effektive wirtschaftspolitische Lösung herbeiführen. Hermann Sautter hat dargestellt, dass eine „einheitliche Weltwirtschaftsordnung“ kritisch bleibt, ${ }^{24}$ weil „funktionsspezifische Regelsysteme, die auf jeweils ein Ziel gerichtet sind, einem Regelsystem überlegen" sind, welches zugleich mehrere Ziele erreichen will (Sautter 2004: 362). ${ }^{25}$ Mit Herms lässt sich diese Einsicht auch so ausdrücken, dass die Teilordnung der Wirtschaft so reguliert werden muss, dass sie eigenständig funktioniert und weder von anderen Funktionsbereichen (z. B. der Politik) überlagert wird noch auf andere übergreift.

Hinzu kommt die Frage: Wenn vom ,Primat der Politik ${ }^{\star}$ und von der Notwendigkeit von Rahmenordnungen gesprochen wird, welche Politik und Ordnung ist gemeint chinesische, arabisch-muslimische, kontinental-europäische oder lateinamerikanische? Die Anerkennung der Bedeutung religiöser und kultureller Ressourcen muss nicht als Kapitulation vor dem ,Kampf der Kulturen' gedeutet werden, die essentialistisch als Großsubjekte stilisiert werden. Stattdessen soll zunächst die Pluralität und Perspektivität von politischen Leitbegriffen und Ordnungen festgestellt werden. Diese Vielfältigkeit der grundlegenden Blickpunkte auf die Welt mit entsprechenden weitreichenden Handlungsfolgen zeichnet sich bereits im relativ kleinen Kreis der wissenschaftlichen Weltdeuter ab (vgl. Prisching 2005) - wie viel mehr im interkulturellen Kontext.

23 In dieser Überzeugung stimmen Herms (2004e) und Homann (2005) überein.

24 Dabei sieht er die Notwendigkeit einer Weltwirtschaftsordnung, in der eine ,möglichst weitgehende Kohärenz der funktionsspezifischen Institutionen“ herrscht (Sautter 2004: 362). Dazu gehören u. a. eine Wettbewerbsordnung für den Güterverkehr, eine Stabilitätsordnung für finanzielle Transaktionen, eine Umweltordnung, eine Sozialordnung für die Verwirklichung von Menschenrechtsstandards und gegen die Marginalisierung von Entwicklungsländern (ebd.: 360f.).

25 Dies entspricht für ihn der ,wirtschaftspolitischen ,Assignment'-Regel, wonach jedes Instrument möglichst nur einem Ziel zugeordnet werden soll“", und er verweist darauf, wie entscheidend es ist, dass die Ressourcenallokation nicht durch ihr fremde Ziele überfordert wird (ebd.). 
In diesem Kontext liegt die Radikalität der Theorie von Herms nicht darin, dass er die Rede von einem Grundkonsens ebenso irreführend findet wie die von dem Weltethos ${ }^{26}$ und der Ethik des Kosmopolitismus ${ }^{27}$ weil es nur vielfältige Ethiken gibt. Vielmehr verdeutlicht sich seine profilierte Position an der These, dass er den dauerhaften weltanschaulichen Widerstreit als Grundlage einer egalitären Ordnung etablieren will. Zunächst komme es auf die Offenlegung des Dissenses der verschiedenen weltanschaulichen und religiösen Positionen an. Wenn diese nicht thematisiert werden, ist laut Herms keine friedliche Koexistenz der Kulturen möglich, weil nicht erkennbar wird, welche Ziele in den einzelnen Funktionsbereichen verfolgt werden oder gar ob die Funktionsbereiche zugunsten autoritärer Systeme aufgelöst werden sollen (vgl. die Beiträge in Herms 2007; 2008). Wir erinnern uns: Herms geht davon aus, dass eine prinzipiell unüberschaubar große Zahl von verschiedenen ethischen Perspektiven besteht. Koexistenz kann für ihn weder durch neutrale Prinzipien noch durch Homogenisierungsanstrengungen erreicht werden. „Erforderlich“ sei vielmehr das „offene Eingeständnis“, dass es einen Konflikt „verschiedener Gesamtlebensgestalten oder ,Kulturen“ mit je eigener weltanschaulicher bzw. religiöser Binnenausrichtung“ gibt. Und erst dann sei die Bereitschaft zum Dialog und zu gegenseitigen Anerkennungsmöglichkeiten zu „erkunden“ (Herms 1998: 345). Die vorschnelle Vereinnahmung in einem wolkigen, indifferenten Globalethos würde Tendenzen eines Kampfes zwischen Kulturen verstärken. Herms geht dagegen von der fundamentalanthropologischen Ausgangsposition aus, dass jede weltanschauliche Position auf Erfahrungen und Überzeugungen und somit auf gesamtgesellschaftlichen Zielen beruht (vgl. Herms 2008). Aus dieser Diagnose erarbeitet er auch die Therapie: Bildung. Alle großen Strebensethiker der Vergangenheit - Herms verweist vor allem auf Adam Smith und David Hume - hätten die „,reale Abhängigkeit des Sachverhalts ,Wirtschaften“ von dem Sachverhalt „Bildung“ durchschaut“ (Herms 2004d: 228). Für ihn stellt sich die Lösung der Koexistenzproblematik ,letztlich“ nicht als politisches oder wirtschaftliches, sondern als Bildungsproblem, „und zwar just und genau“ als Problem der „Bildung der Affekte, des Triebs, des Lebensgefühls, kurz: des Herzens der Menschen“ (Herms 1990: 214).

Im Anschluss an diese Diagnose und Therapie müssten in einer umfangreicheren Studie an Herms Konzeption folgende Fragen bearbeitet werden. ${ }^{28}$ Erstens: Wenn der Widerstreit der Weltanschauungen einerseits so viel radikaler ist als vielfach dargestellt und anderseits eben diese Anschauungen so grundlegend sind für die Gestaltung einer gemeinsamen (Welt-)Gesellschaft, welche institutionellen Formen zur Gestaltung der Koexistenz sind denkbar und vor allem plausibel? Herms setzt auch auf den Dialog der Weltanschauungen, aber gerade der Dialog wird vielerorts verweigert. Insofern

26 Dementsprechend fordert Horst Köhler wiederholt in unterschiedlichsten Kontexten und in Anlehnung an Hans Küng ein Weltethos als Grundkonsens verbindlicher Werte (vgl. Köhler 2009).

$27 \quad$ Ulrich Beck insinuiert solch eine monolithische, kosmopolitische Anerkennungsethik (2007: 340), vgl. auch dieselbe Kritik an Karl Homann bei Herms (2004d: 204) und Grabenstein (1998: 126).

28 Eine solche Forschungsarbeit wird derzeit vom Autor durchgeführt, in der die oben benannte Rationalitätsproblematik vor allem im intergesellschaftlichen Horizont im Mittelpunkt steht. 
muss noch deutlicher herausgearbeitet werden, welche Verfahren interkulturell anerkannt sind, mit denen überhaupt der Widerstreit der Religionen, Ideengebäude und Daseinsgewissheiten zu bändigen ist. Zweitens: Bei Herms bleibt (wie auch insgesamt auf evangelisch-theologischer Seite) unscharf, was mit den angesprochenen Institutionen gemeint ist und welchen genauen Beitrag sie im komplexen Gefüge der Weltöffentlichkeit leisten können. ${ }^{29}$ Seine Forderung nach weltanschaulicher Bildung greift in ein zu Recht höchst umstrittenes Diskussionsfeld ein. Muss nicht doch der religiösen Bildung die Notwendigkeit einer gemeinsamen Grundlage der Sittlichkeit aller Menschen entgegengehalten werden (vgl. v. Hentig 2001)? Schließlich müsste die Konzeption von Herms empirisch fundiert werden. Es ist bemerkenswert, dass Herms Forschungen zu diesem Thema nicht berücksichtigt. So ist von S. M. Eisenstadt und J. P. Arnason das Bild der multiplen Ausprägungen der Moderne entwickelt worden, welches auf dieselbe globalisierte gesellschaftliche Infrastruktur aufbaut, sich aber von allen Hochkulturen gleichermaßen differenziert hat (vgl. Arnason 2003; Habermas 2009: 396ff.). Wenn diese Forschung trägt, könnte einerseits eine Lösung des genannten Verfahrensproblems neu thematisiert und andererseits die interkulturelle Logik von Herms hinterfragt werden - mit dem Hinweis, dass sich nämlich durch globale Infrastruktur auch transkulturelle Überzeugungsformen ausbilden.

\section{Zusammenfassung}

Wir sind ausgegangen von der Auseinandersetzung, ob der Staat oder der Markt die Vorherrschaft in globalen Verträgen einnehmen soll, und haben uns gefragt, ob weltanschauliche Einflüsse in diesen Diskursen hinreichend berücksichtigt worden sind. Eilert Herms stellt zwei weitere Teilordnungen in den Mittelpunkt: den Funktionsbereich der Weltanschauungen und Religionen, in dem gesamtgesellschaftliche, ethische Ziele generiert werden, und den der wissenschaftlichen Interaktion, in dem Wege zu vorher feststehenden Zielen entdeckt werden. Wenn diese zuletzt genannten Bereiche nicht reflektiert, gepflegt und gefördert werden, so sein Ergebnis, werden die fundamentalsten gemeinsamen Handlungen nicht gelingen. Er sensibilisiert insbesondere für die unverfügbaren Erfahrungen und Lebensgefühle, welche die Akteure gesellschaftlicher Prozesse orientieren, indem er verdeutlicht, wie diese Erfahrungen für ihr Wirklichkeitsverständnis und Verhalten konstitutiv sind. Weil sich die Kategorien einer Sozialtheorie und der ethische Erfahrungshorizont ihrer Denker gegenseitig bedingen, sei von einer perspektivischen und kontingenten Theorielage auszugehen. Umso dringlicher ist es danach zu fragen, ob und wie in anderen Kulturen die intergesellschaftliche Koexistenz begründet wird. Die Leistungskraft der Position Herms'

Gerade die evangelische Theologie hat das Thema Globalisierung bisher nur in Ansätzen behandelt. Der außerordentlich hohen Bedeutung des Themas „entspricht merkwürdigerweise keine vergleichbar intensive Bemühung in der Ethik als akademischer Disziplin, jedenfalls im deutschsprachigen Raum“. Durchaus anders sei die Situation ,im Hinblick auf die Urteilsbildung im Raum der Kirchen, insbesondere auf globaler Ebene. An verschiedenen Orten hat sich die ökumenische Bewegung mit dem Thema befasst. (...) Insbesondere aus den Ländern der südlichen Hemisphäre sind eindringliche Beiträge in die weltweite Debatte eingebracht worden. In ihnen äußert sich ein Leidensdruck, der von der Erfahrung der Schattenseiten der Globalisierung geprägt ist" (Bedford-Strohm 2009: 3, vgl. dort auch den Überblick von Konrad Raiser). 
liegt in der radikalen Anerkennung des Pluralismus - der Verständnisse des Nutzens, des Guten, der Pflicht.

Allerdings scheint mir Herms seine berechtigte Pointe zu überziehen. Er will ja in der erfahrungswissenschaftlichen Begründung religiöser Geltungsansprüche nicht nur die Anschlussfähigkeit an die Rationalitätsmodelle der Sozialwissenschaften nachweisen, sondern diese im Horizont jener einordnen. Wenn aber - so ist im Verlauf argumentiert worden - die Reflexionspotenziale der formalen Vernunft derart in materiale Überzeugungen eingebunden werden, ist nicht erkennbar, wie überhaupt ein Diskurs zwischen Geltungsansprüchen stattfinden kann. Argumente und Gründe besitzen ein kritisches Potenzial, das Weltanschauungen als Korrektiv gegenübersteht. Das Verhältnis indes zwischen Kritik und Erschließung ist selbst nicht absolut bestimmbar, sondern dessen Klärung bedarf intersubjektiver, d. h. auch interdisziplinärer und interkultureller Verfahren.

Dass die Religionen eine wesentliche Rolle spielen in der Gestaltung globaler Institutionen, ist verschiedentlich betont worden. Für Herms steht darüber hinaus fest, dass die ökonomischen Interdependenzen und deren politische Regulierung nicht ohne die institutionalisierten Formen der Weltanschauungen und Religionen verstanden und verändert werden können. Seine Position wird dann nicht als Wiedereinführung der Deutungshoheit der Theologen gelesen werden, wenn er mit der von ihm erwünschten subsidiarischen Gesellschaftsordnung Ernst macht, so dass die Religionen ihren begrenzten Einfluss in intersubjektiv kritikfähiger Weise begründen müssen.

\section{Literaturverzeichnis}

Arnason, J. P. (2003): Civilizations in Dispute. Historical Questions and Theoretical Traditions, Leiden: Brill.

Bartling, H./ Luzius, F. (2008): Grundzüge der Volkswirtschaftslehre. Einführung in die Wirtschaftstheorie und Wirtschaftspolitik, 16. Aufl., München: Vahlen.

Beck, U. (2007): Weltrisikogesellschaft. Auf der Suche nach der verlorenen Sicherheit, Frankfurt a. M.: Suhrkamp.

Bedford-Strohm, H. (2009): Vorwort. Systematische Theologie. Globalisierung, in: Verkündigung und Forschung 54, $2 \mathrm{ff}$.

Bhagwati, J. (2007): Verteidigung der Globalisierung (Übers. W. Roller), München: Pantheon.

Feil, E. (2007): Religio. Die Geschichte eines neuzeitlichen Begriffs im 18. und frühen 19. Jahrhundert, Bd. IV (Forschungen zur Kirchen- und Dogmengeschichte 91), Göttingen: Vandenhoeck \& Ruprecht.

Giddens, A. (2007): Europe in the Global Age, Cambridge: Polity Press.

Grabenstein, A. (1998): Wachsende Freiheiten oder wachsende Zwänge? Zur kritischen Wahrnehmung der wachsenden Wirtschaft aus theologisch-sozialethischer Sicht (St. Galler Beiträge zur Wirtschaftsethik), Bern/Stuttgart/Wien: Paul Haupt.

Habermas, J. (2000): Eine Antwort auf die wirtschaftliche Globalisierung. Weltinnenpolitik und weltbürgerliche Solidarität, in: Becher, G./ Treptow, E. (Hrsg.): Die Gerechte Ordnung der Gesellschaft. Texte vom Altertum bis zur Gegenwart, Frankfurt a. M.: Suhrkamp, 331-348. 
Habermas, J. (2009): Die Revitalisierung der Weltreligionen - Herausforderung für ein säkulares Selbstverständnis der Moderne?, in: Habermas, J.: Kritik der Vernunft (Philosophische Texte 5), Frankfurt a. M.: Suhrkamp, 387-408.

Held, D./ Kaya, A. (2008): Global Inequality. Patterns and Explanations, Cambridge: Polity Press.

Hentig, H. v. (2001): Polis und Kosmopolis. „Weltethos“ aus der Sicht eines Pädagogen, in: Küng, H./ Kuschel, K.-J. (Hrsg.): Wissenschaft und Weltethos, München: Piper, 262294.

Herms, E. (1990): Bildung und Ausbildung als Thema der Theologie und Aufgabe der Kirche, in: Herms, E.: Erfahrbare Kirche. Beiträge zur Ekklesiologie, Tübingen: Mohr, 209-221.

Herms, E. (1991): Grundzüge eines theologischen Begriffs sozialer Ordnung, in: Herms, E.: Gesellschaft gestalten, Tübingen: Mohr, 56-94.

Herms, E. (1995): Kirche in der Zeit, in: Herms, E.: Kirche für die Welt. Lage und Aufgabe der evangelischen Kirchen im vereinigten Deutschland, Tübingen: Mohr, 231-317.

Herms, E. (1998): „Eilert Herms“, in: Henning, C./ Lehmkühler, K. (Hrsg.): Systematische Theologie der Gegenwart in Selbstdarstellungen, Tübingen: Mohr, 317-352.

Herms, E. (2003): Menschsein im Werden. Studien zu Schleiermacher, Tübingen: Mohr.

Herms, E. (2004a): Religion und Wirtschaft, in: Herms, E.: Die Wirtschaft des Menschen. Beiträge zur Wirtschaftsethik, Tübingen: Mohr, 1-34.

Herms, E. (2004b): Ethik und Ökonomik. Eine Verhältnisbestimmung, in: ebd.: Die Wirtschaft des Menschen. Beiträge zur Wirtschaftsethik, Tübingen: Mohr, 54-78.

Herms, E. (2004c): Theologische Wirtschaftsethik, in: ebd.: Die Wirtschaft des Menschen. Beiträge zur Wirtschaftsethik, Tübingen: Mohr, 133-162.

Herms, E. (2004d): Normetablierung, Normbefolgung, Normbestimmung. Beobachtungen und Bemerkungen zu Karl Homanns These „Ökonomik - Fortsetzung der Ethik mit anderen Mitteln“, in: ebd.: Die Wirtschaft des Menschen. Beiträge zur Wirtschaftsethik, Tübingen: Mohr, 198-232.

Herms, E. (2004e): ,Gerechte Weltwirtschaftsordnung‘. Begriff und Verwirklichungsbedingungen, in: ebd.: Die Wirtschaft des Menschen. Beiträge zur Wirtschaftsethik, Tübingen: Mohr, 233-252.

Herms, E. (2004f): Globalisierung aus der Sicht christlicher Sozialethik, in: ebd.: Die Wirtschaft des Menschen. Beiträge zur Wirtschaftsethik, Tübingen: Mohr, 253-283.

Herms, E. (2004g): Art. Religion in der Gesellschaft, in: Betz, H. D., et al. (Hrsg.): Religion in Geschichte und Gegenwart. Handwörterbuch für Theologie und Religionswissenschaft, Bd. 7, 4. Aufl., Tübingen: Mohr, Sp. 286-295.

Herms, E. (2005a): Art. Wirtschaftsethik, in: Betz, H. D., et al. (Hrsg.): Religion in Geschichte und Gegenwart. Handwörterbuch für Theologie und Religionswissenschaft, Bd. 8, 4. Aufl., Tübingen: Mohr, Sp. 1621-1624.

Herms, E. (2005b): Art. Vernunft II, III, in: Betz, H. D. et al. (Hrsg.): Religion in Geschichte und Gegenwart. Handwörterbuch für Theologie und Religionswissenschaft, Bd. 8, 4. Aufl., Tübingen: Mohr, 1039-1042.

Herms, E. (2007): Zusammenleben im Widerstreit der Weltanschauungen. Beiträge zur Sozialethik, Tübingen: Mohr.

Herms, E. (2008): Politik und Recht im Pluralismus, Tübingen: Mohr.

Herms, E. (2009): Die Bedeutung der Religion für die Fortentwicklung von Wirtschaft und Gesellschaft, in: Korff, W., et al. (Hrsg.): Wirtschaftsethik. Ein Handbuch, Bd. 1, Berlin: Berlin University Press, 669-682. 
Herms, E./ May, A. (1986ff.): Theologische Aspekte der Wirtschaftsethik. Loccumer Protokolle, 7 Bde., Loccum.

Homann, K. (2005): Globalisierung aus wirtschaftsethischer Sicht, in: Homann, K./ Koslowski, P./ Lütge, C. (Hrsg.): Wirtschaftsethik der Globalisierung, Tübingen: Mohr, 7-15.

Homann, K. (2006): Wirtschaftsethik: Ökonomischer Reduktionismus?, Diskussionspapier 2006-3, Wittenberg Zentrum für globale Ethik, http://www.wcge.org/downloads/DP_2006-3_Homann-

Wirtschaftsethik_oekonomischer_Reduktionismus.pdf (Zugriff am 16.09.10).

Hübner, J. (2009): Eine Weltgesellschaft ist möglich - ein neuer, kritischer Internationalismus ist nötig! Evangelische Sozialethik vor den Herausforderungen des Globalisierungsprozesses, in: Bedford-Strohm, H. et al. (Hrsg.): Globalisierung, Gütersloh: Gütersloher Verlagshaus, 13-33.

Joas, H. (1996): Die Kreativität des Handelns, Frankfurt a. M.: Suhrkamp.

Köhler, H. (2009): „Eine Anleitung zum Überdenken von Gewohnheiten“. Grußwort von Bundespräsident Horst Köhler zur Eröffnung der Podiumsdiskussion „Zukunft der Moderne" am 20. Oktober 2009 in Berlin, http://www.bundespraesident.de/Anlage/original_658680/Grusswort-vonBundespraesident-Horst-Koehler-zur-Eroeffnung-der-Podiumsdiskussion-Zukunftder-Moderne.pdf (Zugriff am 16.09.10).

Lütge, C. (2007): Was hält eine Gesellschaft zusammen? Ethik im Zeitalter der Globalisierung (Die Einheit der Gesellschaftswissenschaften 140), Tübingen: Mohr.

Luhmann, N. (1993): Wirtschaftsethik - als Ethik?, in: Wieland, J. P. (Hrsg.): Wirtschaftsethik und Theorie der Gesellschaft, Frankfurt a. M.: Suhrkamp, 143-147.

Mack, E. (2004): Theologische Wirtschaftsethik, in: Beschorner, Th./ Schmidt, M. (Hrsg.): Integritäts- und Umweltmanagement in der Beratungspraxis (Schriftenreihe für Wirtschafts- und Unternehmensethik 8), München, Mering: Rainer Hampp Verlag, 139-149.

Norris, P./ Inglehart, R. (2004/2007): Sacred and Secular. Religion and Politics Worldwide, Cambridge: Cambridge University Press.

Nuscheler, F./ Messner, D. (2006): Das Konzept Global Governance - Stand und Perspektiven, in: Stiftung Entwicklung und Frieden (Hrsg.): Sonderband: Global Governance für Entwicklung und Frieden. Perspektiven nach einem Jahrzehnt, Bonn: Dietz Verlag, 1879 .

Prisching, M. (2005): Die Globalisierung von zeitdiagnostischen Wirtschafts- und Gesellschaftsmodellen, in: Homann, K./ Koslowski, P./ Lütge C. (Hrsg.): Wirtschaftsethik der Globalisierung, Tübingen: Mohr, 34-61.

Radermacher, F.-J. (2005): Balance oder Zerstörung. Ökosoziale Marktwirtschaft als Schlüssel zu einer weltweiten nachhaltigen Entwicklung, 4. Aufl., Wien: Ökosoziales Forum.

Riesebrodt, M. (2001): Die Rückkehr der Religionen. Fundamentalismus und der „Kampf der Kulturen“, 2. Aufl., München: Beck.

Rittberger, V. (2003): Weltregieren, in: Küng, H./ Senghaas, D. (Hrsg.): Friedenspolitik. Ethische Grundlagen internationaler Beziehungen, München: Piper, 175-208.

Sassen, S. (2008): Das Paradox des Nationalen. Territorium, Autorität und Rechte im globalen Zeitalter (Übers. N. Gramm), Frankfurt a. M.: Suhrkamp.

Sautter, H. (2004): Weltwirtschaftsordnung. Die Institutionen der globalen Ökonomie, München: Franz Vahlen.

Stiglit, J. (2008): Die Chancen der Globalisierung (Übers. Th. Schmidt), München: Pantheon. 
Sundermeier, T. (2007): Was ist Religion? Religionswissenschaft im theologischen Kontext, 2. erw. Aufl., Frankfurt a. M.: Lembeck.

Tofall, N. (2005): Die Eigenart der Güter. Eilert Herms macht die Ökonomik wieder zur Magd der Theologie, in: Frankfurter Allgemeine Zeitung, Nr. 55, 07.03.05, 14.

Ulrich, P. (2008): Integrative Wirtschaftsethik. Grundlagen einer lebensdienlichen Ökonomie, 4. Aufl., Bern: Haupt. 